\title{
Lung injury caused by paraquat poisoning results in increased interleukin-6 and decreased microRNA-146a levels
}

\author{
WEI WU ${ }^{1 *}$ and YONG LI ${ }^{2 *}$ \\ ${ }^{1}$ Department of Respiratory Medicine; ${ }^{2}$ Emergency Department, Affiliated Hospital of \\ Jining Medical University, Jining, Shandong 272029, P.R. China
}

Received June 4, 2017; Accepted January 3, 2018

DOI: $10.3892 /$ etm.2018.6153

\begin{abstract}
The aim of the present study was to investigate the expression of microRNA (miR)-146a in the pulmonary macrophages, peripheral blood mononuclear cells and serum of patients with lung injury caused by paraquat poisoning, as well as the underlying mechanism of its regulation in the disease. A total of 26 patients with lung injury caused by paraquat poisoning were included in the present study. In addition, 33 healthy subjects were included as the control group. The expression levels of interleukin (IL)-6 mRNA and miR-146a was determined by reverse transcription-quantitative polymerase chain reaction (RT-qPCR). Western blotting was used to measure IL-6 protein expression, while enzyme-linked immunosorbent assay was also performed to determine the secretion of IL- 6 protein. A dual-luciferase reporter assay was conducted to examine whether IL-6 mRNA is a direct target of miR-146a. Patients with lung injury caused by paraquat poisoning exhibited higher IL-6 mRNA and protein levels as compared with those in healthy subjects. In addition, miR-146a expression in patients with paraquat poisoning-induced lung injury was significantly reduced in comparison with that in healthy subjects. Notably, the overexpression of miR-146a by mimic transfection downregulated the expression of IL-6 in pulmonary macrophages. The results of dual-luciferase reporter assay demonstrated that IL-6 mRNA was a direct target of miR-146a. Therefore, the present study demonstrated that increased expression of IL-6 in patients with lung injury caused by paraquat poisoning is associated with decreased expression of miR-146a. Furthermore, miR-146a may regulate the occurrence and immune response of lung injury caused by
\end{abstract}

Correspondence to: Dr Yong Li, Emergency Department, Affiliated Hospital of Jining Medical University, 89 Guhuai Road, Jining, Shandong 272029, P.R. China

E-mail: lywwjn@126.com

*Contributed equally

Key words: microRNA-146a, lung injury, paraquat poisoning, interleukin-6 paraquat poisoning and this process is possibly achieved via IL-6, an important cytokine that mediates inflammation.

\section{Introduction}

Paraquat is a type of bipyridine compound that damages the majority of internal organs following ingestion, particularly the heart, liver, lungs and kidney (1-3). Due to its wide range of usage, it has become the herbicide with the highest acute poisoning mortality rate (4). The high mortality rates of paraquat caused by incorrect intake or suicidal oral ingestion have been a huge challenge for health care workers.

In organs damaged by paraquat poisoning, the lungs present the most evident inflammatory characteristics at an early stage, including impaired alveolar epithelial cells, intra-alveolar hemorrhage and edema, inflammatory cell infiltration, and irreversible fibrosis in the alveoli and pulmonary interstitium (5). Anti-inflammatory treatments are involved in the majority of early treatment regimens for paraquat poisoning. In addition, certain key genes with large differences in expression during the occurrence and development of inflammation have become candidate targets for gene therapy.

Interleukin (IL)-6, a type of lymphokine produced by activated monocytes and tissue macrophages, is an important factor in immune response. IL-6 transforms B-cell precursors into cells that produce antibodies, promote the growth and differentiation of primary bone marrow-derived cells, and enhance the lysis function of natural killer cells (6-8). To date, studies investigating the role of mRNA and microRNA (miRNA or miR) molecules in the regulation mechanism of IL-6 have achieved promising results. For instance, miR-365 was observed to negatively regulate IL-6 expression in 293 cells and HeLa cells (9). However, to the best of our knowledge, studies on the regulation and upstream miRNA of IL-6 in lung injury due to paraquat poisoning have not been conducted. miR-146a is a member of the miR-146 family, which is primarily involved in the regulation of inflammation and the innate immune system (10). To the best of our knowledge miR-146a has not been previously investigated in association with paraquat poisoning and its association with IL-6 has not been examined (11).

In the present study, the expression levels of IL-6 mRNA and protein in the macrophages, peripheral blood mononuclear cells (PBMCs) and serum of patients with paraquat 
poisoning-induced lung injury were determined, and the association between IL-6 and miR-146a was investigated.

\section{Materials and methods}

Patients. A total of 26 patients with lung injury caused by paraquat poisoning who received treatments at the Affiliated Hospital of Jining Medical University (Jining, China) between August 2013 and February 2017 were included in the present study. In addition, 33 healthy subjects who undertook physical examinations at this hospital in the same period were included into the control group. Blood and alveolar lavage fluid were collected from all patients and healthy subjects. Among the 26 patients with lung injury caused by paraquat poisoning, 16 were males and 10 were females (age range, 18-56 years; median age, 39 years). In the control group, 18 individuals were males and 15 were females (age range, 20-58; median age, 41 years). Patients with lung injury caused by paraquat poisoning did not present complications or infection in the heart, liver or kidney, and did not suffer from immune or immune-associated diseases, such as diabetes and tumors. Subjects in the control group requested fiber bronchoscopy due to symptoms including foreign body sensation at pharynx or chest tightness. None of the healthy subjects in the control group exhibited abnormal results in the pulmonary function, chest X-ray and fiber bronchoscopy, or were smokers. All procedures were approved by the Ethics Committee of Jining Medical University (Jining, China). Written informed consents were obtained from all patients or their families.

Samples. To collect pulmonary macrophages, alveolar lavage was initially performed 4-6 times ( $20 \mathrm{ml}$ each time) at the bronchial opening. A total of 40-60\% fluid was recollected, filtered (100 $\mu \mathrm{m}$ pore size) and centrifuged at $1,000 \mathrm{x}$ for $10 \mathrm{~min}$ at $4^{\circ} \mathrm{C}$. Next, the precipitates were subjected to cytological classification according to a previously published method (12) and the cell density was adjusted to $1-3 \times 10^{6}$ cells $/ \mathrm{ml}$ in PBS. Phthalocyanine blue (Guangzhou Chemical Reagent Factory, Guangzhou, China) staining was conducted at room temperature for $3 \mathrm{~min}$, indicating that up to $90 \%$ cells were viable. All cells were seeded onto clean glass slides and cultured in Dulbecco's modified Eagle's medium (Thermo Fisher Scientific, Inc., Waltham, MA, USA) at $37^{\circ} \mathrm{C}$ for $60 \mathrm{~min}$. Subsequent to washing off any floating cells using $0.5 \mathrm{~mol} / 1$ PBS, the remaining adherent cells were macrophages, which were stored at $-20^{\circ} \mathrm{C}$. To examine whether miR-146a expression altered the expression of IL-6, pulmonary macrophages were transfected with either $100 \mathrm{nM}$ agomiR-negative control (NC; forward, 5'-UAAACGGGUGACAGGUUUUAUC-3' and reverse, 5'-GAUAAAUCCUGUCACCCGUUUA-3') or $100 \mathrm{nM}$ agomiR-146a (forward, 5'-UGAGAACUGAAUUCC AUGGGUU-3' and reverse, 5'-AACCCAUGGAAUUCAGUU CUCA-3'; Guangzhou RiboBio Co., Ltd., Guangzhou, China) using Lipofectamine ${ }^{\circledR} 2000$ (Life Sciences; Thermo Fisher Scientific, Inc.), according to the manufacturer's protocol. Following $48 \mathrm{~h}$ the transfected cells were used for subsequent experiments.

Peripheral blood (10-15 ml) was collected from all participants and stored at $4^{\circ} \mathrm{C}$ for $1-2 \mathrm{~h}$. Next, the serum was separated by centrifugation at $400 \mathrm{x} \mathrm{g}$ at $4^{\circ} \mathrm{C}$ for $10 \mathrm{~min}$, and aliquots were added into Eppendorf tubes (100 $\mu 1$ in each tube) prior to storage at $-70^{\circ} \mathrm{C}$.

In order to collect PBMCs, anticoagulant venous blood was mixed with an equal volume of serum-free Iscove's modified Dulbecco's medium (Thermo Fisher Scientific, Inc.) and the mixture was added onto the surface of human lymphocyte separation solution ( $5 \mathrm{ml}$; Cedarlane, Burlington, ON, Canada). Following centrifugation at $400 \mathrm{x}$ g for $30 \mathrm{~min}$, the middle mist layer was gently aspirated into tubes, and mixed with 5 times of volumes of Hank's solution (Beijing Solarbio Science \& Technology Co., Ltd., Beijing, China), followed by centrifugation at $300 \mathrm{x} g$ for $10 \mathrm{~min}$. Subsequent to washing twice with PBS, the cells were diluted into a density of $1 \times 10^{6} / \mathrm{ml}$, and a total of $3 \times 10^{6}$ cells were seeded into culture dishes (bottom area, $9 \mathrm{~cm}^{2}$ ). After cultivation at $37^{\circ} \mathrm{C}$ and under $5 \% \mathrm{CO}_{2}$ for $2 \mathrm{~h}$, the cells that adhered at the bottom of the dishes were identified as PBMCs by microscopic evaluation. Non-adhered cells were washed off with PBS and PBMCs were trypsinized and collected for further use.

Reverse transcription-quantitative polymerase chain reaction (RT-qPCR). Total RNA was extracted from $100 \mu \mathrm{l}$ liquid samples or $3 \times 10^{6}$ cells using TRIeasy ${ }^{\mathrm{TM}}$ reagent following the manufacturer's protocol (cat. no. 10606ES60; Yeasen Biotechnology Co., Ltd., Shanghai, China). The concentration and quality of RNA was measured using ultraviolet spectrophotometry (Nanodrop ND2000; Thermo Fisher Scientific, Inc., Waltham, MA, USA). Subsequently, cDNA was obtained by RT from $1 \mu \mathrm{g}$ RNA and stored at $-20^{\circ} \mathrm{C}$. The RT of mRNA was performed using the TIANScript II cDNA First Strand Synthesis kit (KR107; Tiangen Biotech Co., Ltd., Beijing, China), and RT of miRNA was conducted using the miRcute miRNA cDNA First Strand Synthesis kit (KR201; Tiangen Biotech Co., Ltd.).

In order to detect the mRNA expression of IL-6, the SuperReal PreMix (SYBR Green) RT-qPCR kit (FP204; Tiangen Biotech Co., Ltd.) was used, with GAPDH serving as an internal reference. The primer sequences were as follows: IL-6, 5'-GGCACTGGCAGAAAACAACC-3' (forward) and 5'-GCAAGTCTCCTCATTGAATCC-3' (reverse); GAPDH, 5'-GGGAAACTGCGGCGTGAT-3' (forward) and 5'-AAA GGTGGAGGAGTGGGT-3' (reverse). The reaction system (20 $\mu \mathrm{l}$ ) was composed of $10 \mu \mathrm{l}$ RT-qPCR Mix, $0.5 \mu 1$ upstream

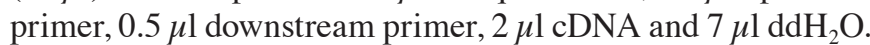
The qPCR conditions involved an initial denaturation at $95^{\circ} \mathrm{C}$ for $30 \mathrm{sec}$, followed by 45 cycles of denaturation at $95^{\circ} \mathrm{C}$ for $5 \mathrm{sec}$ and elongation at $57^{\circ} \mathrm{C}$ for $30 \mathrm{sec}$ (iQ5; Bio-Rad Laboratories, Inc., Hercules, CA, USA). The $2^{-\Delta \Delta \mathrm{Cq}}$ method (13) was used to calculate the relative expression of IL- 6 mRNA against GAPDH. Each sample was tested in triplicate.

The expression of miR-146a was determined by miRcute miRNA RT-PCR Kit (FP401; Tiangen Biotech Co., Ltd.), using U6 as an internal reference. The primer sequences used were as follows: miR-146a, 5'-CGGCGGTGAGAACTGAATTCC A-3' (forward) and 5'-GTGCAGGGTCCGAGGT-3' (reverse); U6, 5'-CTCGCTTCGGCAGCACA-3' (forward) and 5'-AAC GCTTCACGAATTTGCGT-3' (reverse). The reaction system (20 $\mu \mathrm{l}$ ) consisted of $10 \mu \mathrm{l}$ RT-qPCR Mix, $0.5 \mu \mathrm{l}$ upstream primer, $0.5 \mu \mathrm{l}$ downstream universal primer, $2 \mu \mathrm{l}$ cDNA and $7 \mu \mathrm{lddH_{2 }}$ O. The reaction protocol involved an initial 
denaturation at $95^{\circ} \mathrm{C}$ for $5 \mathrm{~min}$, followed by 40 cycles of denaturation at $95^{\circ} \mathrm{C}$ for $10 \mathrm{sec}$, annealing at $60^{\circ} \mathrm{C}$ for $20 \mathrm{sec}$ and elongation at $72^{\circ} \mathrm{C}$ for $10 \mathrm{sec}$ (iQ5; Bio-Rad Laboratories, Inc.). The $2^{-\Delta \Delta \mathrm{Cq}}$ method was used to calculate the relative expression of miR-146a against U6. Each sample was tested in triplicate.

Western blotting. According to a previously published study (14), precooled radioimmunoprecipitation assay lysis buffer $[600 \mu \mathrm{l}$; containing $50 \mathrm{mM}$ Tris, $1 \mathrm{mM}$ EDTA, $150 \mathrm{mM} \mathrm{NaCl}, 0.1 \%$ sodium dodecyl sulfate (SDS), $1 \%$ Triton $\mathrm{X}-100$ and $1 \%$ sodium deoxycholate; Beyotime Institute of Biotechnology, Shanghai, China] was used to lyse PBMCs for $50 \mathrm{~min}$ on ice. Next, the mixture was centrifuged at $12,000 \mathrm{x} \mathrm{g}$ at $4^{\circ} \mathrm{C}$ for $5 \mathrm{~min}$. The protein concentration of the obtained supernatant was determined by a bicinchoninic acid protein concentration determination kit (RTP7102; Real-Times Biotechnology Co., Ltd., Beijing, China). Protein samples $(20 \mu \mathrm{g})$ were then mixed with SDS loading buffer, followed by denaturation in boiling water bath for $5 \mathrm{~min}$, and were then subjected to $10 \%$ SDS-polyacrylamide gel electrophoresis. The resolved proteins were transferred to polyvinylidene difluoride membranes on ice for $2 \mathrm{~h}$ at $100 \mathrm{~V}$ and blocked with 5\% skimmed milk for $1 \mathrm{~h}$ at room temperature. Subsequently, the membranes were incubated overnight at $4^{\circ} \mathrm{C}$ with the following primary antibodies: Rabbit anti-human polyclonal IL-6 antibody (1:1,000; ab6672; Abcam, Cambridge, UK) and rabbit anti-human $\beta$-actin antibody (1:5,000; ab129348; Abcam). Following extensive washing with PBS with Tween 20 three times for $15 \mathrm{~min}$ each, the membranes were incubated with goat anti-rabbit horseradish peroxidase-conjugated secondary antibody (1:3,000; ab6721; Abcam) at room temperature for $1 \mathrm{~h}$. The samples were further washed with PBS with Tween 20 three times for $15 \mathrm{~min}$ each, and then the membrane was developed with an enhanced chemiluminescence detection kit (ab65623; Abcam). Image Lab version 3.0 software (Bio-Rad Laboratories, Inc.) was used to acquire and analyze imaging signals. The relative content of IL- 6 protein was expressed in terms of the IL- $6 / \beta$-actin ratio.

Enzyme-linked immunosorbent assay (ELISA). According to a previously published study (12), the IL-6 level in the serum obtained from patients and healthy controls was tested using the human IL-6 ELISA kit (ab178013; Abcam, Cambridge, UK). Briefly, standards (50 $\mu \mathrm{l})$, samples (10 $\mu \mathrm{l}$ sample liquid and $40 \mu \mathrm{l}$ diluent) and blank were added to predefined wells in 96-well microplates. In the standard and sample wells, horseradish peroxidase-labelled conjugates $(100 \mu \mathrm{l})$ were added prior to sealing the plates for incubation at $37^{\circ} \mathrm{C}$ for $1 \mathrm{~h}$. Subsequent to washing the plates five times with wash solution provided in the kit, substrates $\mathrm{A}(50 \mu \mathrm{l})$ and $\mathrm{B}(50 \mu \mathrm{l})$ were added into each well. After incubation at $37^{\circ} \mathrm{C}$ for $15 \mathrm{~min}$, stop solution $(50 \mu \mathrm{l})$ was added into each well, and the absorbance of the wells was measured at $450 \mathrm{~nm}$ within 15 min using a Multiskan $^{\text {TM }}$ FC microplate reader (Thermo Fisher Scientific, Inc.).

Bioinformatics analysis and dual-luciferase reporter assay. Bioinformatics prediction is a powerful tool for the investigation of the functions of miRNAs. Thus, to understand the regulatory mechanism of IL-6, various databases were used to predict miRNA molecules that may regulate IL-6, including miRanda (www.microrna.org/microrna/home.do), TargetScan (www.targetscan.org), PiTa (genie.weizmann. ac.il/pubs/mir07/mir07_data.html), RNAhybrid (bibiserv. techfak.uni-bielefeld.de/rnahybrid) and PicTar (pictar. mdc-berlin.de/). It was identified that miR-146a was able to potentially regulate IL-6 (Fig. 1).

According to the bioinformatics results, wild-type (WT) and mutant (MT) seed regions of miR-146a in the 3'-untranslated region (UTR) of IL-6 gene were chemically synthesized in vitro, the Spe-1 and Hind III restriction sites were added, and then the regions were cloned into pMIR-REPORT luciferase reporter plasmids. According to a previously published study (13), plasmids $(0.8 \mu \mathrm{g})$ with WT or MT 3'-UTR DNA sequences were co-transfected with $100 \mathrm{nM}$ agomiR-NC (forward, 5'-UAAACGGGUGACAGGUUUUAUC-3' and reverse, 5'-GAUAAAUCCUGUCACCCGUUUA-3') or $100 \mathrm{nM}$ agomiR-146a mimics (forward, 5'-UGAGAACUG AAUUCCAUGGGUU-3' and reverse, 5'-AACCCAUGGAAU UCAGUUCUCA-3'; Guangzhou RiboBio Co., Ltd.) into 293T cells (The Cell Bank of Type Culture Collection of Chinese Academy of Sciences, Shanghai, China) using Lipofectamine $2000^{\circledR}$ (Thermo Fisher Scientific, Inc.). After cultivation for $48 \mathrm{~h}$ the cells were lysed using a dual-luciferase reporter assay kit (Promega Corp., Fitchburg, WI, USA) according to the manufacturer's protocol, and the fluorescence intensity was measured using GloMax 20/20 luminometer (Promega Corp.). Using the Renilla fluorescence activity as an internal reference, the fluorescence values of each group of cells were measured.

Statistical analysis. The results were analyzed using SPSS version 18.0 statistical software (IBM Corp., Armonk, NY, USA). The data are expressed as the means \pm standard deviation. Data were tested for normality, and multigroup measurement data were analyzed using one-way analysis of variance. In the case of homogeneity of variance, the least significant difference and Student-Newman-Keuls methods were used. In the case of heterogeneity of variance, Tamhane's T2 or Dunnett's T3 method was used. $\mathrm{P}<0.05$ was considered to indicate a statistically significant difference.

\section{Results}

Patients with paraquat poisoning-induced lung injury exhibit higher IL-6 mRNA levels compared with healthy subjects. To measure the expression of IL-6 mRNA in different samples, RT-qPCR was conducted. The data revealed that the levels of IL-6 mRNA in the pulmonary macrophages, PBMCs and serum of patients with lung injury caused by paraquat poisoning were all significantly higher when compared with those in the control group $(\mathrm{P}<0.05$; Fig. 2A-C).

IL-6 protein expression in macrophages and PBMCs is upregulated in patients with lung injury caused by paraquat poisoning. To determine the expression of IL-6 protein in macrophages and PBMCs, western blotting was performed. The data demonstrated that the IL- 6 protein expression levels in macrophages and PBMCs from patients with lung injury caused by paraquat poisoning were significantly elevated 
3' uuggguaccuuaaguCAAGAGu 5' hsa-miR-146a

||||||

\section{8:5' cacagaacuuauguugUUCUCu 3' IL-6}

Figure 1. Direct interaction between miR-146a and IL- 6 was detected by bioinformatics prediction. In order to examine the regulatory mechanism of IL-6, the databases miRanda, TargetScan, PiTa, RNAhybrid and PicTar were used to predict miRNA molecules that may regulate IL-6, and it was observed that miR-146a was able to potentially regulate IL-6. miR, microRNA; IL-6, interleukin-6.
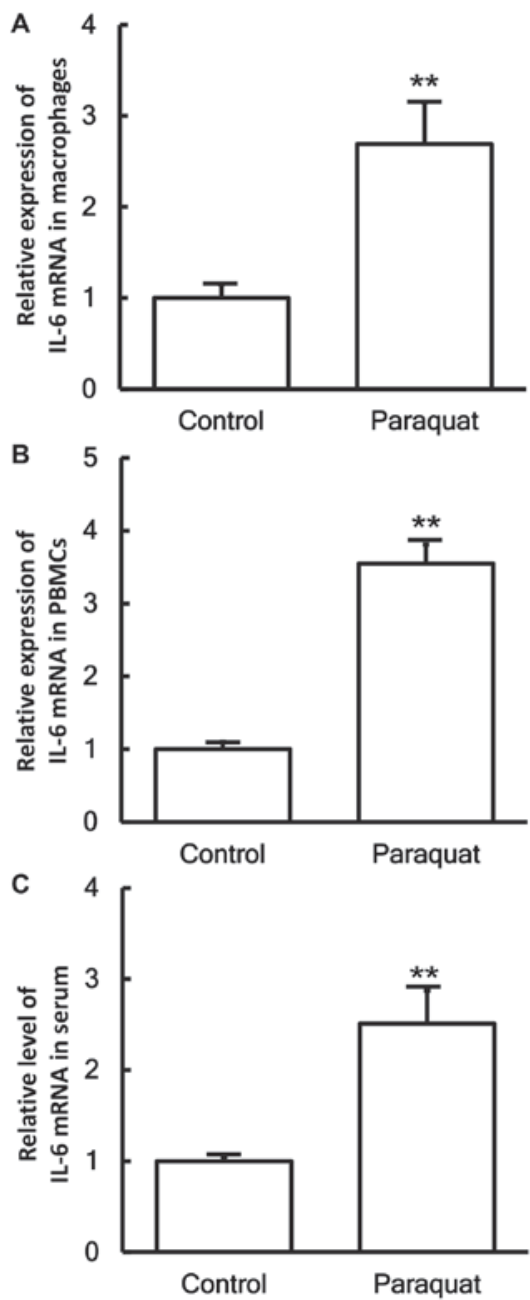

Figure 2. Expression levels of IL-6 mRNA in (A) pulmonary macrophages, (B) PBMCs and (C) serum obtained from healthy control subjects and patients with lung injury caused by paraquat poisoning, as determined by reverse transcription-quantitative polymerase chain reaction. ${ }^{* *} \mathrm{P}<0.01$ vs. control group. IL-6, interleukin-6; PBMCs, peripheral blood mononuclear cells.

compared with those obtained from healthy subjects $(\mathrm{P}<0.05$; Fig. 3A and B).

Higher serum IL-6 content in patients with lung injury caused by paraquat poisoning. To examine the secretion of IL- 6 in the blood, ELISA was performed. The data indicated that the IL-6 content in the serum of patients with lung injury caused by paraquat poisoning was significantly increased as compared with that in healthy subjects $(\mathrm{P}<0.05$; Fig. 4).
Reduced miR-146a levels in patients with lung injury caused by paraquat poisoning. To investigate the levels of miR-146a in the cells and serum, RT-qPCR was employed. The data demonstrated that the expression levels of miR-146a in the macrophages, PBMCs and serum of patients with lung injury caused by paraquat poisoning were significantly decreased in comparison with those in healthy subjects $(\mathrm{P}<0.05$; Fig. 5A-C).

miR-146a expression downregulates the expression of IL-6 in pulmonary macrophages. To examine whether miR-146a expression affects the expression of IL-6, pulmonary macrophages were transfected with agomiR-146a and then subjected to RT-qPCR and western blotting. The results revealed that the levels of miR-146a in cells transfected with agomiR-146a were significantly higher in comparison with those in the negative control group $(\mathrm{P}<0.05$; Fig. 6A). In addition, the expression levels of IL-6 mRNA and protein in cells transfected with agomiR-146a were significantly lower as compared with those in the negative control group (both $\mathrm{P}<0.05$; Fig. $6 \mathrm{~B}$ and $\mathrm{C}$ ). These results suggest that miR-146a expression downregulates the expression of IL-6 in pulmonary macrophages.

miR-146a binds to the 3'-UTR seed region of IL-6 mRNA to regulate its expression. To identify the interaction between miR-146a and the 3'-UTR of IL-6 mRNA, a dual-luciferase reporter assay was performed. The fluorescence value of cells co-transfected with miR-146a mimics and pMIR-REPORT-WT luciferase reporter plasmids was significantly reduced when compared with that of cells transfected with miR-NC $(\mathrm{P}<0.05)$. By contrast, the fluorescence value of cells co-transfected with miR-146a mimics and pMIR-REPORT-MT luciferase reporter plasmids was not significantly different from that of cells transfected with miR-NC ( $\mathrm{P}>0.05$; Fig. 7). These results indicate that miR-146a is able to bind to the 3'-UTR seed region of IL-6 mRNA in order to regulate its expression.

\section{Discussion}

Paraquat has been registered as a type of herbicide since 1962, and is widely used to date (15), and it is the second most used compound among all herbicides (16). However, paraquat poisoning has been reported to result in a high fatality rate (4), with no specific antidote currently known for this poisoning. One of the characteristics of early paraquat poisoning is a strong inflammatory reaction. IL-6 is an inflammatory factor that has been extensively studied. It induces the production of C-reactive proteins and fibrinogen in inflammation, and promotes thrombosis (17). Increased levels of IL-6 in the body may cause inflammatory diseases, including rheumatoid arthritis and Crohn's disease, due to binding to the IL-6 receptors (18). In rheumatoid arthritis, IL-6 stimulates the secretion of inflammation mediators by $\mathrm{T}$ lymphocytes and $\mathrm{B}$ lymphocytes, facilitates the maturation and differentiation of B lymphocytes, and increases the effects of IL-1 $\beta$ and tumor necrosis factor $\alpha$ (TNF- $\alpha)$. In inflammation, IL-6 presents chemotaxis to other inflammatory cells, such as neutrophilic lymphocytes and mononuclear macrophages (19). These observations suggest that IL-6 serves important roles in inflammation responses. In the present study, it was demonstrated that the expression levels of IL-6 mRNA and protein 
A
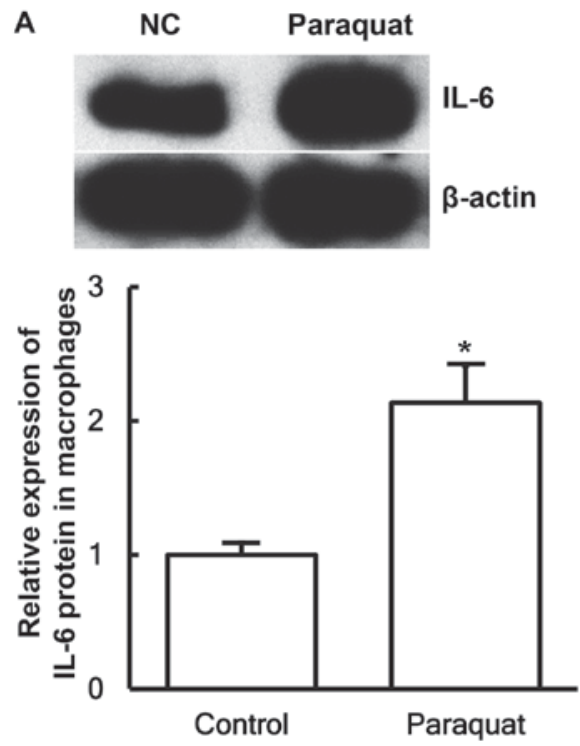

B
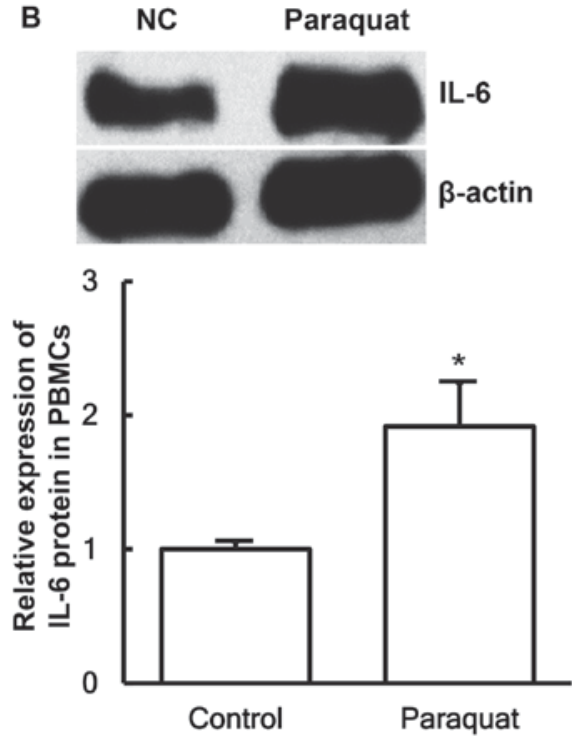

Figure 3. Expression of IL-6 protein in (A) pulmonary macrophages and (B) PBMCs obtained from healthy control subjects and patients with lung injury caused by paraquat poisoning, examined by western blotting. "P<0.05 vs. control group. IL-6, interleukin-6; PBMCs, peripheral blood mononuclear cells.

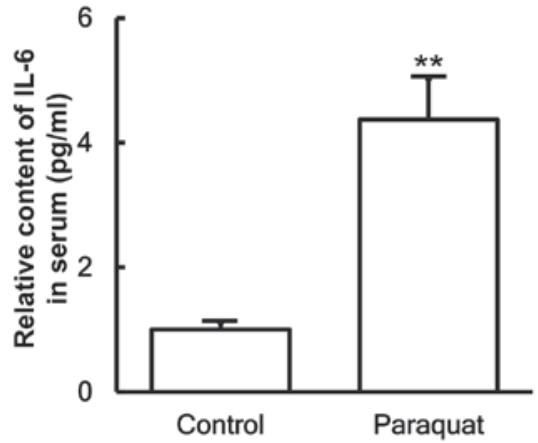

Figure 4. Content of IL-6 protein in the serum of healthy control subjects and patients with lung injury caused by paraquat poisoning, as determined by enzyme-linked immunosorbent assay. ${ }^{* *} \mathrm{P}<0.01$ vs. control group. IL-6, interleukin-6.

were upregulated in the pulmonary macrophages, PBMCs and serum of patients with lung injury caused by paraquat poisoning, consistent with the inflammatory characteristics of early lung injury.

miRNA widely participates in various pathophysiological processes, including the proliferation, invasion and migration of tumor cells, hypertension, diabetes mellitus and atherosclerosis $(20,21)$. It has been reported that expression of miR-146a is abnormal in autoimmune diseases, such as rheumatoid arthritis $(22,23)$. Clinical and animal models of osteoarthritis revealed that miR-146a is associated with pain in osteoarthritis (24-26). Furthermore, multiple nuclear factor- $\kappa \mathrm{B}(\mathrm{NF}-\kappa \mathrm{B})$ binding sites exist in the promoter region of the miR-146a gene, while lipopolysaccharide, IL-1 and TNF- $\alpha$ promote the expression of miR-146a in an NF-кB-dependent manner (27-30). In the present study, bioinformatics analysis demonstrated that miR-146a and IL-6, two genes that are closely involved in inflammation, may have a regulatory association, and that IL- 6 may be a direct target gene of miR-146a. The results also demonstrated
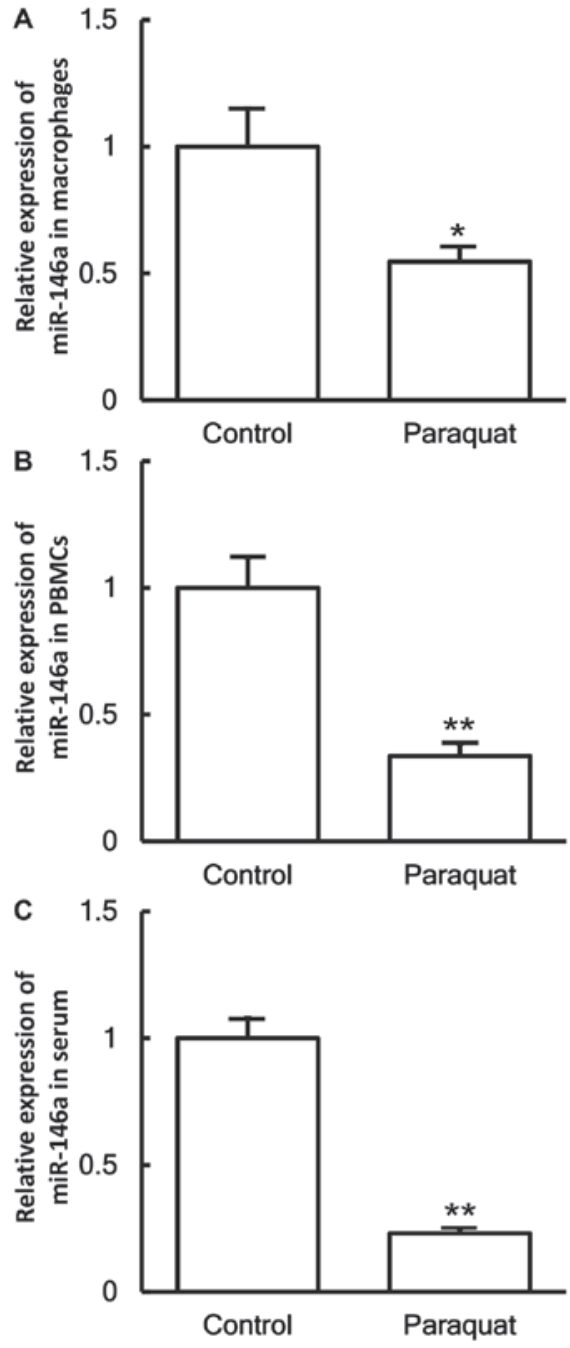

Figure 5. Expression of miR-146a in (A) pulmonary macrophages, (B) PBMCs and $(C)$ serum obtained from healthy control subjects and patients with lung injury caused by paraquat poisoning, examined by reverse transcription-quantitative polymerase chain reaction. ${ }^{*} \mathrm{P}<0.05$ and ${ }^{* *} \mathrm{P}<0.01$ vs. control group. miR, microRNA; PBMCs, peripheral blood mononuclear cells. 
A

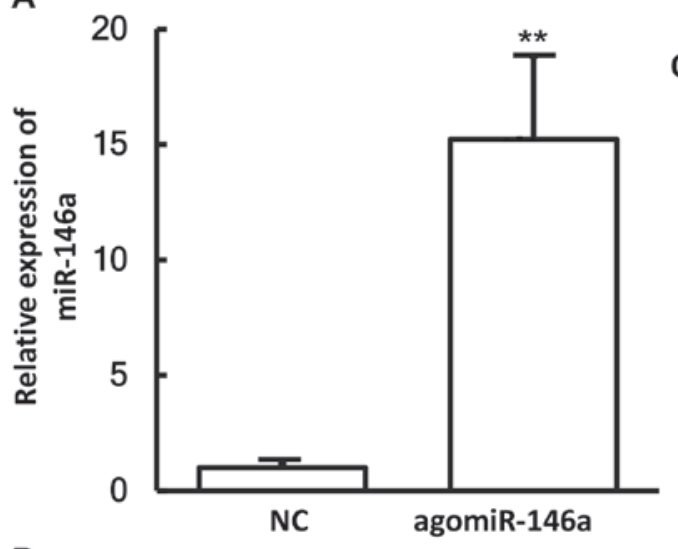

C

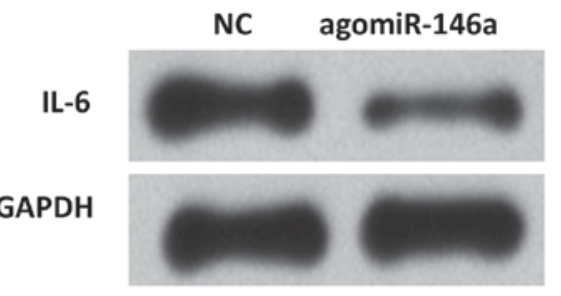

B
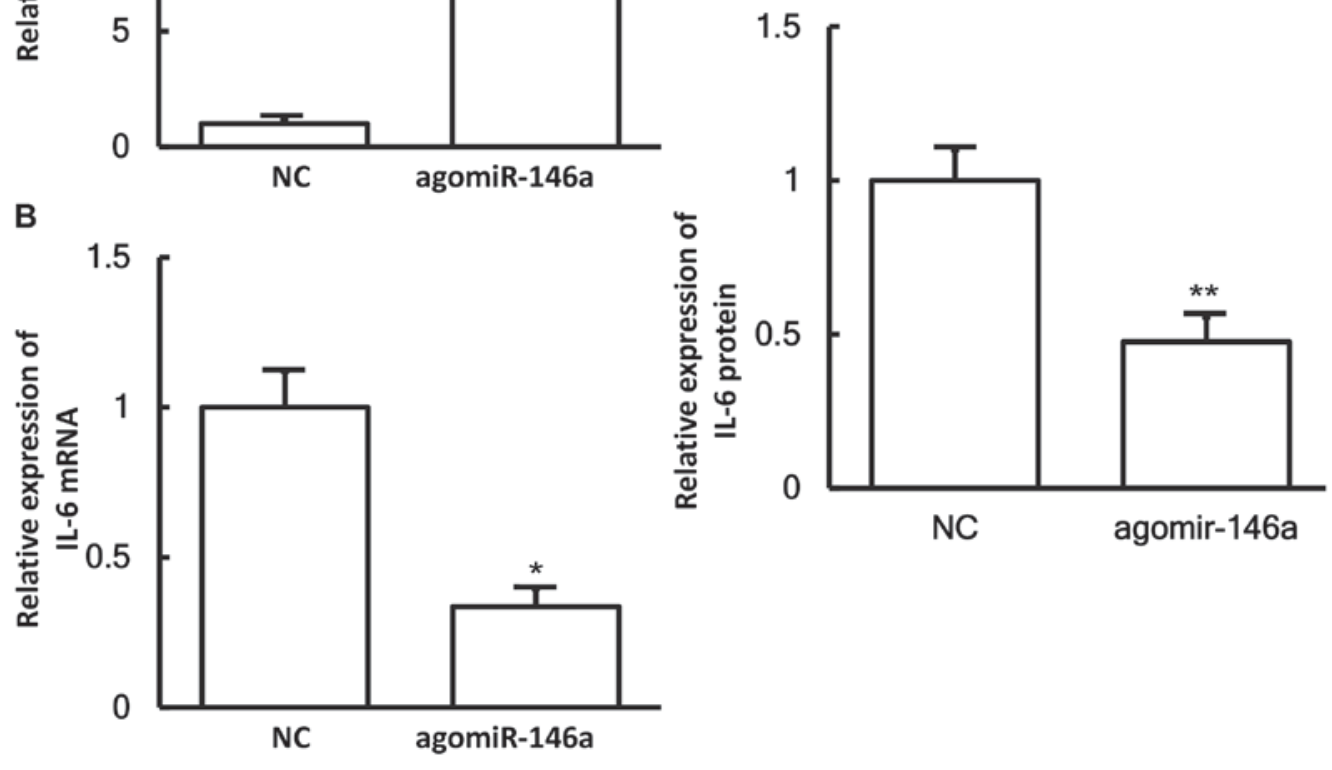

Figure 6. Effect of miR-146a on IL-6 expression. The expression levels of (A) miR-146a, (B) IL-6 mRNA and (C) IL-6 protein in pulmonary macrophages transfected with agomiR-146a. Reverse transcription-quantitative polymerase chain reaction was used to measure the expression of miR-146a and IL-6 mRNA, while western blotting was performed to determine the expression of IL- 6 protein. ${ }^{*} \mathrm{P}<0.05$ and ${ }^{* *} \mathrm{P}<0.01$ vs. NC group. miR, microRNA; NC, negative control.

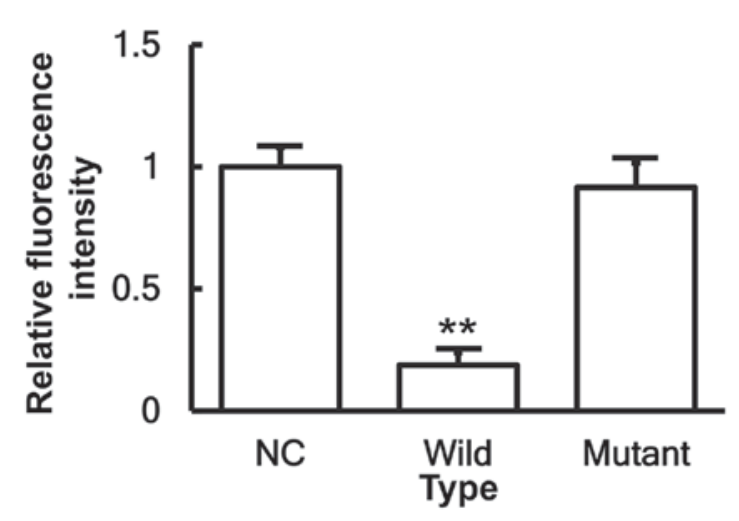

Figure 7. Identification of interaction between miR-146a and IL-6 using dual-luciferase reporter assay. Plasmids $(0.8 \mu \mathrm{g})$ with wild-type or mutant 3'-untranslated region DNA sequences were co-transfected with miR-146a mimics into 293T cells. After cultivation for $24 \mathrm{~h}$, the cells were subjected to dual-luciferase reporter assay, and the fluorescence intensity was measured, with the Renilla fluorescence activity serving as an internal reference. ${ }^{* *} \mathrm{P}<0.01$ vs. NC group. miR, microRNA; NC, negative control.

that miR-146a was downregulated and IL-6 was upregulated in pulmonary macrophages and PBMCs, suggesting that the immune system of the body negatively regulated the cleavage of IL- 6 by miR-146a and promoted immune responses by enhancing the expression of IL-6. In addition, reduced expression of miR-146a and enhanced expression of IL-6 were detected in the serum, indicating that these serum levels may reflect the inflammation responses and tissue damages in lung injury caused by paraquat poisoning. Finally, a dual-luciferase reporter assay demonstrated that IL-6 was a direct target gene of miR-146a, since overexpression of miR-146a reduced the fluorescence intensity of the IL-6 luciferase reporter plasmid.

In conclusion, the present study demonstrated that the increased expression of IL-6 in patients with lung injury caused by paraquat poisoning was associated with a decreased expression of miR-146a. A limitation of the present study is the small sample size, which should be increased in future studies. In addition, additional experiments should be designed to test the effect of paraquat on other cell types or in an animal model. The present study provides a basis for the development of novel treatments for lung injury caused by paraquat poisoning.

\section{Acknowledgements}

The authors would like to thank Dr Luning Jiang from the Department of Respiratory Medicine, Affiliated Hospital of Jining Medical University (Jining, China).

\section{Funding}

No funding was received. 


\section{Availability of data and materials}

The datasets used and/or analyzed during the current study are available from the corresponding author on reasonable request.

\section{Authors' contributions}

YL designed the study and analyzed the data. WW performed the experiments and analyzed the data.

\section{Ethics approval and consent to participate}

All procedures were approved by the Ethics Committee of Jining Medical University (Jining, China). Written informed consents were obtained from all patients or their families.

\section{Consent for publication}

All patients provided written informed consent for the publication of their data.

\section{Competing interests}

The authors confirm that they have no competing interests.

\section{References}

1. Mullick FG, Ishak KG, Mahabir R and Stromeyer FW: Hepatic injury associated with paraquat toxicity in humans. Liver 1: 209-221, 1981.

2. Nagao M, Takatori $T$, Inoue $K$, Shimizu M, Terazawa $K$ and Akabane H: Immunohistochemical localization and dynamics of paraquat in small intestine, liver and kidney. Toxicology 63 : 167-182, 1990.

3. Dinis-Oliveira RJ, Remião F, Duarte JA, Ferreira R, Sánchez Navarro A, Bastos ML and Carvalho F: P-glycoprotein induction: An antidotal pathway for paraquat-induced lung toxicity. Free Radic Biol Med 41: 1213-1224, 2006.

4. Hwang KY, Lee EY and Hong SY: Paraquat intoxication in Korea. Arch Environ Health 57: 162-166, 2002.

5. Yamashita M, Yamashita M and Ando Y: A long-term follow-up of lung function in survivors of paraquat poisoning. Hum Exp Toxicol 19: 99-103, 2000

6. Anderson AE, Pratt AG, Sedhom MA, Doran JP, Routledge C, Hargreaves B, Brown PM, Lê Cao KA, Isaacs JD and Thomas R: IL-6-driven STAT signalling in circulating CD4+ lymphocytes is a marker for early anticitrullinated peptide antibody-negative rheumatoid arthritis. Ann Rheum Dis 75: 466-473, 2016.

7. Tezono K, Sarker KP, Kikuchi H, Nasu M, Kitajima I and Maruyama I: Bioactivity of the vascular endothelial growth factor trapped in fibrin clots: Production of IL-6 and IL-8 in monocytes by fibrin clots. Haemostasis 31: 71-79, 2001.

8. Vila N, Reverter JC, Yagüe J and Chamorro A: Interaction between interleukin- 6 and the natural anticoagulant system in acute stroke. J Interferon Cytokine Res 20: 325-329, 2000.

9. Xu Z, Xiao SB, Xu P, Xie Q, Cao L, Wang D, Luo R, Zhong Y, Chen HC and Fang LR: miR-365, a novel negative regulator of interleukin- 6 gene expression, is cooperatively regulated by $\mathrm{Sp} 1$ and NF-kappaB. J Biol Chem 286: 21401-21412, 2011.

10. Sonkoly E, Ståhle M and Pivarcsi A: MicroRNAs and immunity: Novel players in the regulation of normal immune function and inflammation. Semin Cancer Biol 18: 131-140, 2008.

11. Ye EA and Steinle JJ: miR-146a suppresses STAT3/VEGF pathways and reduces apoptosis through IL-6 signaling in primary human retinal microvascular endothelial cells in high glucose conditions. Vision Res 139: 15-22, 2017.
12. Livak KJ and Schmittgen TD: Analysis of relative gene expression data using real-time quantitative PCR and the 2(-Delta Delta C(T)) method. Methods 25: 402-408, 2001

13. Stjärne Aspelund A, Hammarström $\mathrm{H}$, Inghammar M, Larsson $\mathrm{H}$, Hansson L, Christensson B and Påhlman LI: Heparin-binding protein, lysozyme, and inflammatory cytokines in bronchoalveolar lavage fluid as diagnostic tools for pulmonary infection in lung transplanted patients. Am J Transplant 18: 444-452, 2018.

14. He X, Ping J and Wen D: MicroRNA-186 regulates the invasion and metastasis of bladder cancer via vascular endothelial growth factor C. Exp Ther Med 14: 3253-3258, 2017.

15. Wu H, Song $\mathrm{J}$ and $\mathrm{Ma} \mathrm{J}$ : Paraquat is approved for continued use in the European Union. Pest Sci Administ 25: 36-37, 2004.

16. Yamamoto M, Toda M, Tanaka K, Sugita T, Sasaki S, Uneyama C and Morikawa K: Study on usage of pesticides in various countries. Kokuritsu Iyakuhin Shokuhin Eisei Kenkyusho Hokoku: 92-100, 2007.

17. Tang YH, Vital S, Russell J, Seifert $\mathrm{H}$ and Granger DN: Interleukin-6 mediates enhanced thrombus development in cerebral arterioles following a brief period of focal brain ischemia. Exp Neurol 271: 351-357, 2015.

18. Suzuki Y, Matsui T, Ito H, Ashida T, Nakamura S, Motoya S, Matsumoto T, Sato N, Ozaki K, Watanabe M and Hibi T: Circulating interleukin 6 and albumin, and infliximab levels are good predictors of recovering efficacy after dose escalation infliximab therapy in patients with loss of response to treatment for crohn's disease: A prospective clinical trial. Inflamm Bowel Dis 21: 2114-2122, 2015 .

19. Schaper F and Rose-John S: Interleukin-6: Biology, signaling and strategies of blockade. Cytokine Growth Factor Rev 26: 475-487, 2015

20. Liz J and Esteller M: lncRNAs and microRNAs with a role in cancer development. Biochim Biophys Acta 1859: 169-176, 2016.

21. Varshney J and Subramanian S: MicroRNAs as potential target in human bone and soft tissue sarcoma therapeutics. Front Mol Biosci 2: 31, 2015

22. Abou-Zeid A, Saad M and Soliman E: MicroRNA 146a expression in rheumatoid arthritis: Association with tumor necrosis factor-alpha and disease activity. Genet Test Mol Biomarkers 15: 807-812, 2011.

23. Pauley KM, Satoh M, Chan AL, Bubb MR, Reeves WH and Chan EK: Upregulated miR-146a expression in peripheral blood mononuclear cells from rheumatoid arthritis patients. Arthritis Res Ther 10: R101, 2008.

24. Li X, Kroin JS, Kc R, Gibson G, Chen D, Corbett GT, Pahan K, Fayyaz S, Kim JS, van Wijnen AJ, et al: Altered spinal microRNA-146a and the microRNA-183 cluster contribute to osteoarthritic pain in knee joints. J Bone Miner Res 28: 2512-2522, 2013

25. Li X, Gibson G, Kim JS, Kroin J, Xu S, van Wijnen AJ and Im HJ: MicroRNA-146a is linked to pain-related pathophysiology of osteoarthritis. Gene 480: 34-41, 2011.

26. Yamasaki K, Nakasa T, Miyaki S, Ishikawa M, Deie M, Adachi N, Yasunaga Y, Asahara $\mathrm{H}$ and Ochi M: Expression of MicroRNA-146a in osteoarthritis cartilage. Arthritis Rheum 60: 1035-1041, 2009.

27. Larner-Svensson HM, Williams AE, Tsitsiou E, Perry MM, Jiang X, Chung KF and Lindsay MA: Pharmacological studies of the mechanism and function of interleukin-1beta-induced miRNA-146a expression in primary human airway smooth muscle. Respir Res 11: 68, 2010.

28. Cameron JE, Yin Q, Fewell C, Lacey M, McBride J, Wang X, Lin Z, Schaefer BC and Flemington EK: Epstein-Barr virus latent membrane protein 1 induces cellular MicroRNA miR-146a, a modulator of lymphocyte signaling pathways. J Virol 82: 1946-1958, 2008.

29. Taganov KD, Boldin MP, Chang KJ and Baltimore D: NF-kappaB-dependent induction of microRNA miR-146, an inhibitor targeted to signaling proteins of innate immune responses. Proc Natl Acad Sci USA 103: 12481-12486, 2006.

30. Curtale G, Citarella F, Carissimi C, Goldoni M, Carucci N, Fulci V, Franceschini D, Meloni F, Barnaba V and Macino G: An emerging player in the adaptive immune response: microRNA-146a is a modulator of IL-2 expression and activation-induced cell death in T lymphocytes. Blood 115: 265-273, 2010. 